\title{
Influence of Dynamics and Trajectory on Integrated GPS/INS Navigation Performance
}

\author{
J. Wang, H.K. Lee, S. Hewitson and Hyung-Keun Lee \\ The University of New South Wales, Sydney NSW 2052 Australia \\ Received: 20 November 2003 / Accepted: 28 December 2003
}

\begin{abstract}
The integrated GPS/INS system has become an indispensable tool for providing precise and continuous position, velocity, and attitude information for many positioning and navigation applications. Therefore, it is important to gain insights into the characteristics of the integrated GPS/INS system performance, particularly their relationships with key operational factors, such as the trajectory and dynamics. Such knowledge can be used to improve the quality of positioning and navigation results from integrated GPS/INS systems. In order to analyse the influence of vehicle dynamics and trajectory, simulation and field tests have been carried out in this research. The test results show that the vehicle dynamic changes significantly affect the Kalman filter initialisation time and estimation performance depending on the system operational environments.
\end{abstract}

Key words: Integrated GPS/INS system, Navigation, Vehicle dynamics and trajectory

\section{Introduction}

Global Positioning System (GPS) and Inertial Navigation System (INS) have complementary operational characteristics (e.g, Savage, 2000). It is well known that INS can provide a complete set of navigation parameters with a short-term stability due to the error characteristics of its sensor components. Thus, the accuracy of a standalone INS deteriorates very rapidly with time, which can be compensated by GPS. Integrating GPS with INS can arguably leverage the best of each component system. The advantages of GPS/INS integration, relative to either GPS or INS only, are reported to be a high data rate of complete navigation solutions (e.g., position, velocity, and attitude) with a consistent long-term accuracy, improved availability, smoother trajectories, and greater integrity (Farrell and Barth, 1998; Greenspan, 1996).
Hence, these systems have been used for a wide range of applications, for instance aerial photogrammetry and gravimetry, mobile mapping, vehicle navigation, guidance and control (see e.g., Bevely et al., 2000; Da et al., 1997; Grejner-Brzezinska et al., 1998b; Kwon and Jekeli, 2001; Wang et al., 2003).

Integrated GPS/INS can be implemented using a Kalman filter in different modes, such as loosely, tightly and ultra-tightly coupled. In these integration modes, the INS sensor error states, together with all navigation error states and other unknown parameters of interest, are estimated using a dynamic model and GPS measurements such as Doppler, pseudo-ranges, and/or carrier phases. It has been reported from the literature that the vehicle dynamic and trajectory changes can improve the Kalman filter estimation performance (e.g., Bar-Itzhack \& Porzt, 1980; Porzt \& Bar-Itzhack, 1981; Hong et al., 2002; Wang et al., 2003). The improvement can be described by the fact that the error model becomes time-varying nature that enhances the filter observability. Hence, it is necessary to get insights into the characteristics of the integrated GPS/INS positioning and navigation performance, particularly their relationships with the vehicle trajectory and dynamic changes.

This paper will study how the vehicle dynamics and trajectory changes influence on the performance of an integrated GPS/INS system through both simulation and real data analyses. The optimisation of trajectories and dynamics during system initialisation and operational navigation mode, and in the event of GPS signal blockages, will be discussed.

\section{Error Model and Covariance Analysis}

\subsection{Strapdown INS (SDINS) error model}

In order to study the behaviour of an inertial navigation system, an appropriate presentation error model is necessary. The description of the INS error propagation 
using a linearised error model has been widely used to derive the characteristics of INS error behaviour. A number of the different models can be found in the literature. Among them, the Bar-Itzhack and Berman's mode (Bar-Itzhack and Berman, 1988) is adopted in this research. As the model is designed for Gimballed INS, modification is made through including the coordinate transformation matrix between body and navigation frames to drive the Strapdown INS (SDINS) model. Note that a local level coordinate system (NED: North, East, Down) is used as a navigation frame. Hence, the SDINS error model can be described as follows:

$$
\begin{aligned}
& \dot{x}=A x+w, w \sim N(0, Q) \\
& {\left[\begin{array}{c}
\dot{x}_{r} \\
\dot{x}_{v} \\
\dot{x}_{\psi} \\
\dot{x}_{\nabla} \\
\dot{x}_{\varepsilon}
\end{array}\right]=\left[\begin{array}{c:c:c:c:c}
\Omega & I & 0 & 0 & 0 \\
\hdashline F_{21} & \widetilde{\Omega} & F_{j} & C & 0 \\
\hdashline 0 & 0 & \Omega & 0 & -C \\
\hdashline 0 & 0 & 0 & F_{44} & 0 \\
\hdashline 0 & 0 & 0 & 0 & F_{55}
\end{array}\right]\left[\begin{array}{l}
x_{r} \\
x_{v} \\
x_{\psi} \\
x_{\nabla} \\
x_{\varepsilon}
\end{array}\right]+\left[\begin{array}{c}
w_{r} \\
w_{v} \\
w_{\psi} \\
w_{\nabla} \\
w_{\varepsilon}
\end{array}\right]}
\end{aligned}
$$

where, $I$ and 0 are the third-order identity and zero matrices; $C$ is the coordinate transformation matrix between the body and navigation frames; $w_{r \sim \varepsilon}$ are all zero-mean Gaussian white noise vectors; $x_{r}, x_{v}, x_{\psi}$, $x_{\nabla}$, and $x_{\varepsilon}$ are, respectively, the position, velocity, attitude, accelerometer, and gyro measurement error vectors. The error states included in these vectors are as follows:

$$
\begin{aligned}
& x_{r}=\left[\delta r_{N}, \delta r_{E}, \delta r_{D}\right]^{T} \\
& x_{v}=\left[\delta v_{N}, \delta v_{E}, \delta v_{D}\right]^{T} \\
& x_{\psi}=\left[\delta \psi_{N}, \delta \psi_{E}, \delta \psi_{D}\right]^{T} \\
& x_{\nabla}=\left[\delta \nabla_{x}, \delta \nabla_{y}, \delta \nabla_{z}\right]^{T} \\
& x_{\varepsilon}=\left[\delta \varepsilon_{x}, \delta \varepsilon_{y}, \delta \varepsilon_{z}\right]^{T}
\end{aligned}
$$

where $\nabla$ is accelerometer bias and $\varepsilon$ is gyro drift. Both two accelerometer bias and gyro drift are modeled as first order Gaussian-Markov processes here.

The details of $A$ matrix are given below:

$$
\Omega=\left[\begin{array}{ccc}
0 & \Omega_{D} & -\Omega_{E} \\
-\Omega_{D} & 0 & \Omega_{N} \\
\Omega_{E} & -\Omega_{N} & 0
\end{array}\right]
$$

where

$$
\begin{aligned}
& \Omega_{N}=\dot{\lambda} \cos L \\
& \Omega_{E}=-\dot{L} \\
& \Omega_{D}=-\dot{\lambda} \sin L
\end{aligned}
$$$$
\bar{\Omega}=\left[\begin{array}{ccc}
0 & \bar{\Omega}_{D} & -\bar{\Omega}_{E} \\
-\bar{\Omega}_{D} & 0 & \bar{\Omega}_{N} \\
\bar{\Omega}_{E} & -\bar{\Omega}_{N} & 0
\end{array}\right]
$$

with

$$
\begin{aligned}
& \bar{\Omega}_{N}=(\omega+\dot{\lambda}) \cos L \\
& \bar{\Omega}_{E}=\quad-\dot{L} \\
& \bar{\Omega}_{D}=-(\omega+\dot{\lambda}) \sin L \\
& \widetilde{\Omega}=\left[\begin{array}{ccc}
0 & \widetilde{\Omega}_{D} & -\widetilde{\Omega}_{E} \\
-\widetilde{\Omega}_{D} & 0 & \widetilde{\Omega}_{N} \\
\widetilde{\Omega}_{E} & -\widetilde{\Omega}_{N} & 0
\end{array}\right]
\end{aligned}
$$

where

$$
\begin{aligned}
& \widetilde{\Omega}_{N}=(2 \omega+\dot{\lambda}) \cos L \\
& \widetilde{\Omega}_{E}=c--\dot{L} \\
& \widetilde{\Omega}_{D}=-(2 \omega+\dot{\lambda}) \sin L \\
& F_{\mathrm{j}}=C\left[\begin{array}{ccc}
0 & -f_{z} & f_{y} \\
f_{z} & 0 & -f_{x} \\
-f_{y} & f_{x} & 0
\end{array}\right] \\
& F_{21}=\operatorname{diag}\left[-\frac{g}{R_{n}},-\frac{g}{R_{n}}, \frac{2 g}{R_{n}}\right] \\
& F_{44}=\operatorname{diag}[-\xi,-\xi,-\xi] \\
& F_{55}=\operatorname{diag}[-\beta,-\beta,-\beta]
\end{aligned}
$$

in which $\omega$ is the Earth rate vector; $L$ and $\lambda$ respectively denotes latitude and longitude; $f_{x}, f_{y}$, and $f_{z}$ are the measurements sensed by an accelerometer; $g$ is the gravity value; $R_{n}$ represents the 
radius of the parallel curvature; $\xi$ and $\beta$ are $1 /$ (correlation time) of accelerometer bias and gyro drift processes, respectively.

\subsection{Covariance analysis}

Covariance analysis is a common tool to provide numerical time histories depicting the accuracy of a given configuration in terms of the covariance of its associated error state vector (Savage, 2000). Hence, the analysis can be used to evaluate the performance of the suboptimal filter that operates in a real world environment, and can be utilized as a basic design tool during the synthesis and test of the suboptimal configuration, which is typically based on a simplified error state dynamic/measurement model.

Formulation of linear covariance equation with respect to a system for which feedback the optimally estimated state vector for control reset is (Savage, 2000; Maybeck, 1979):

$$
\begin{aligned}
P_{k}^{c}(+)= & \left\{I-D K_{f} H_{k}\right\} P_{k}^{e}\left\{I-D K_{f} H_{k}\right\}^{T} \\
& +D K_{f} R K_{f}^{T} D^{T}
\end{aligned}
$$

where, $c$ denotes the application of control resets; $e$ is the application of estimation resets; $D$ denotes the feedback matrix

In this study, a suboptimal filter consisting of 17-sates is designed to estimate the INS navigation and sensor errors and feedback them to the INS input (control reset) with respect to a designed real world system (true) model comprising 71 error states. Note that all the analyses will be conducted using a GPS/INS integration system based on GPS pseudo-range observations and a tactical-grade

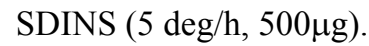

\section{Simulation Study}

In order to investigate the influence of vehicle dynamics and trajectory on the GPS/INS integration system performance, a serious of covariance simulation analyses are carried out.

\subsection{Effect of vehicle dynamics on filter estimation}

The observability of a linear system represents the possibility of determining the state variables using the information on the input and the output of a system. One of the reasons for considering the observability of a dynamic system is the need to determine the efficiency of a Kalman filter that estimates the states of that system.
A covariance simulation was carried out to investigate comprehensive GPS/SDINS system observability (e.g, performance) due to the fact that the error covariance of Kalman filter is one of the indices to check the degree of observability (Ham and Brown, 1983). A trajectory used in the simulation comprised four segments: a constant velocity manoeuvring for 600 seconds, an accelerating with $0.167 \mathrm{~m} / \mathrm{sec}^{2}$ for 100 seconds, a 90 degree turning with angular velocity of $1.8 \mathrm{deg} / \mathrm{sec}$, and a constant velocity moving for 100 seconds.

Figure 1 shows the covariance simulation results for twelve states in the integration filter, except for three position states, which are directly observable from the measurements. The figure indicates that heading error, horizontal accelerometer bias and vertical gyro drift have poor estimation performance (e.g., poor observability) during the first constant velocity manoeuvring, when compared with other states. However, it can also be seen from the figure that the estimation performance of these four states is improved, when the vehicle dynamics are changed (e.g., acceleration and 90 angle turn).
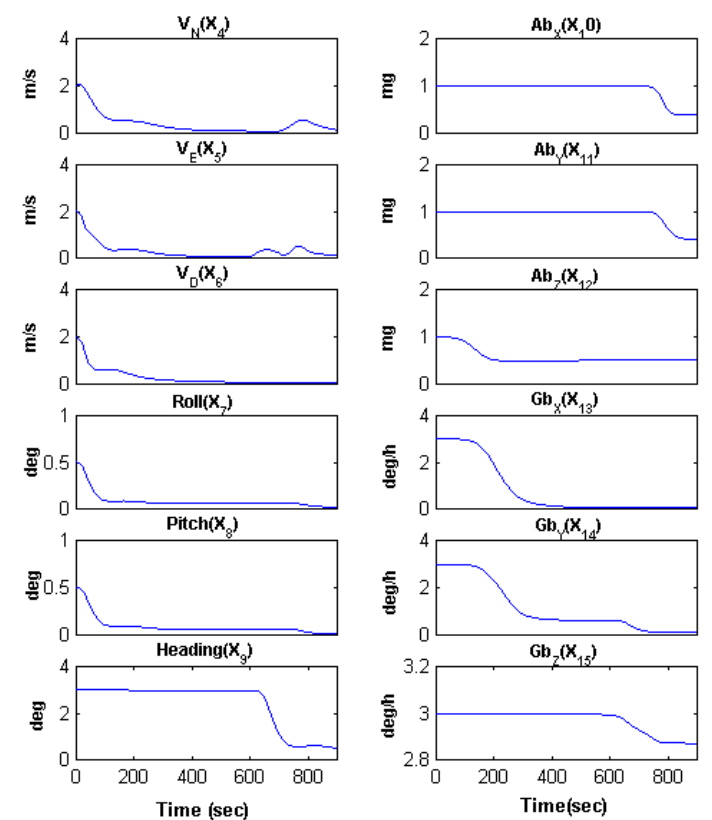

Fig. 1 Covariance simulation results for four dynamic segments

In order to study how different vehicle trajectories and dynamics affect filter estimation performance, further simulations analyses were conducted with respect to the states that have poor observability. In these analyses, four different trajectories (defined as 'Circle', 'Line', 'Rectangle', 'S-turn') were considered. Their plane trajectories and velocity changes are depicted in Fig. 2. Note that the first two segments (e.g., stationary mode for 300 seconds and accelerating with $0.45 \mathrm{~m} / \mathrm{sec}^{2}$ for 40 seconds) are commonly considered in all the trajectory generations, then the vehicle moves according to the 
characteristics of each trajectory. Hence, all the simulation analyses were carried out for 3600 seconds. When taking a look at Figure 2, the characteristics of each trajectory can be described as:

- Circle: the heading angle is continuously changed along clock-wise direction;

- Line: moving with the constant heading and North velocity (e.g., 0 degree and $18 \mathrm{~m} / \mathrm{sec}$, respectively);

- Rectangle: a 90 degree turn is made every 50 seconds;

- S-turn: the vehicle turns are continuously made along clock-wise and counter clock-wise directions.
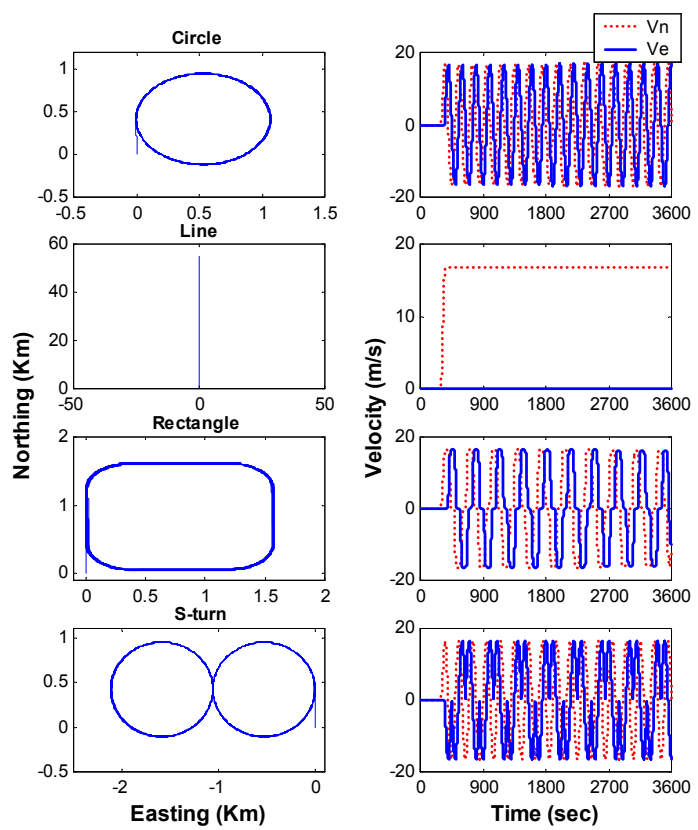

Fig. 2 Four different trajectories for the simulations

Figs. 3 to 5 show the noise variances of some crucial and less observable states (i.e., heading error and horizontal accelerometer biases). In these figures, the top graphs show the results from epoch 1 to epoch 800, whereas the bottom graphs illustrate those from epoch 801 to epoch 360. It is possible to draw the following conclusions from these results: (a) the filter performance can be improved by steady-turning ('Circle', 'Rectangle', 'S-turn' cases) when compared with the 'Line' (constant-velocity), (b) the quickest filter initialisation of the three states is achieved with the 'S-turn'; c) the performance of heading error estimation of the 'Circle', 'Rectangle' and 'S-turn' becomes similar; and d) the 'Circle' trajectory provides the best estimation performance in the horizontal accelerometer biases.
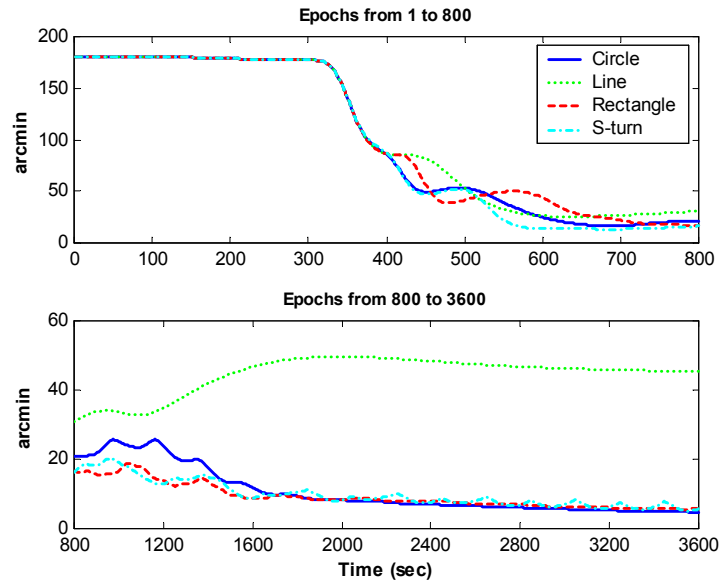

Fig. 3 Covariance simulation results for the heading error state
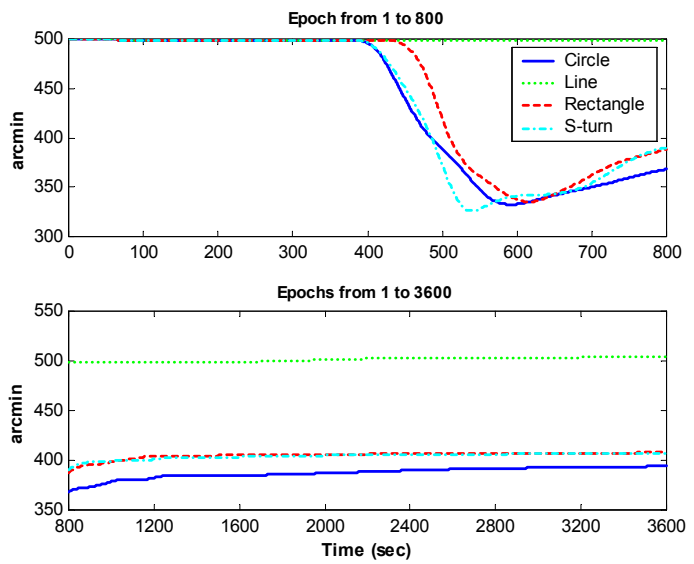

Fig. 4 Covariance simulation results for the horizontal accelerometer bias ( $\mathrm{x}$-axis)
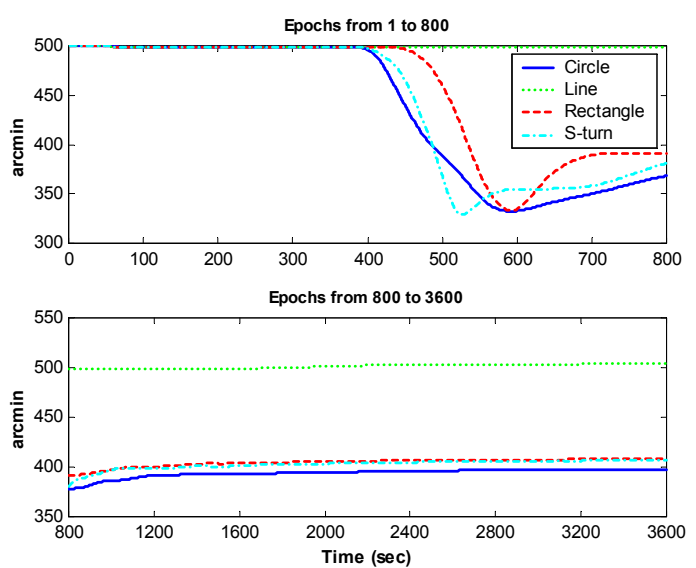

Fig. 5 Covariance simulation results for the horizontal accelerometer bias (y-axis)

\subsection{Navigation error behavior during a GPS blockage}

Further simulation analyses were carried out to study the effect of vehicle dynamics and trajectory on navigation 
error estimation in the Kaman filter during GPS outages. The scenarios for the two tests are as follows (Fig. 6):

- The vehicle remained in stationary mode for 600 seconds before moving in circles within the same trajectory for 680 seconds ('Circle');

- The vehicle stayed in static mode for 600 seconds before moving in circles within the same trajectory for 400 seconds, then in a straight line at a constant speed for 280 seconds ('Line').

The GPS blockage was simulated for the last 280 seconds. It is also important to note that all the navigation and sensor error states in the filter reach the steady-state condition before GPS signals are blocked. Fig. 7 shows the covariance simulation results, which indicate error behaviours of navigation parameters (e.g., position, velocity, and attitude). Note that Circular Error Probability (CEFP) and Probable Error (PE) in the figure represent horizontal and vertical positioning errors, respectively. These results indicate that all the navigation errors rapidly deteriorate according to the length of time that GPS is unavailable. Among these results, the most interesting is the position and heading error behaviour, since the difference in the error increase between the two trajectories are relatively large (e.g., around 15 meters in position, and 10 arc-minutes in heading). Comparing these results, those obtained from the 'Line' are superior to the 'Circle'. This is opposite to the result obtained in the preceding section. Therefore, it can be noted that the vehicle's lateral acceleration changes enhance the integration filter's performance when the filter can be continuously updated by external measurements, whereas it degrades the integrated GPS/INS navigation performance during the GPS blockage.

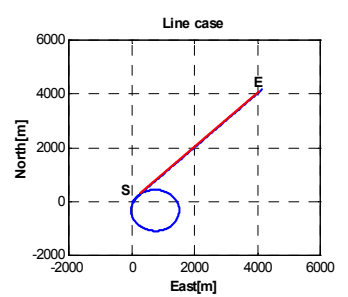

$$
\begin{aligned}
& \text { S: Starting Point } \\
& \text { E: Ending Point }
\end{aligned}
$$
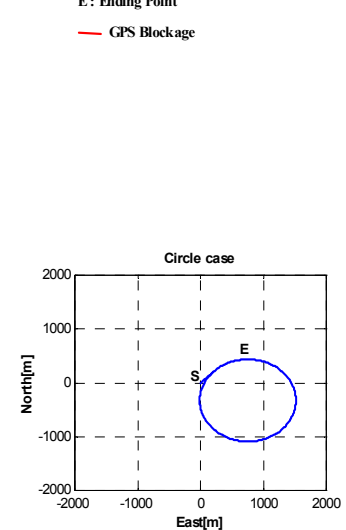

Fig. 6 Trajectories used in the GPS blockage simulation
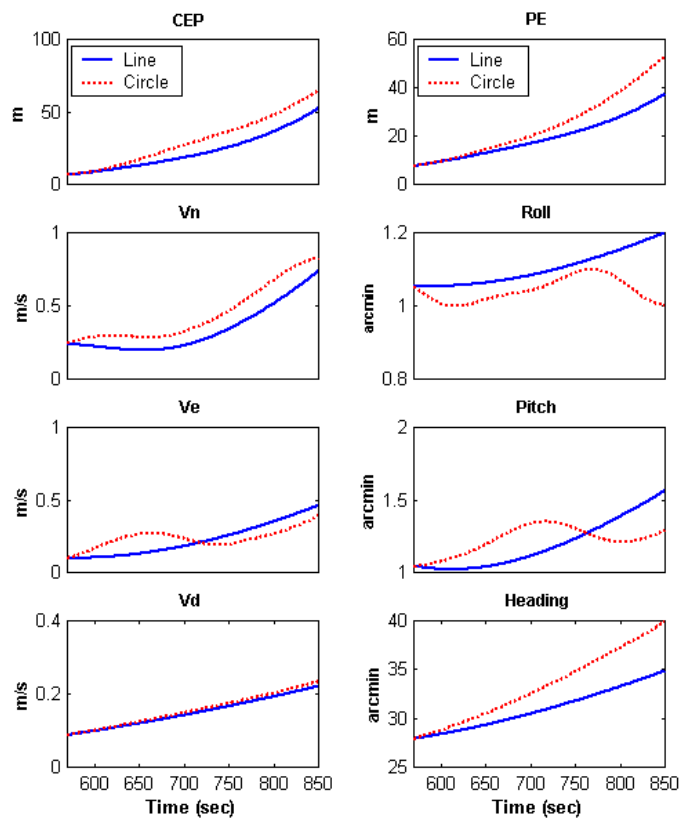

Fig. 7 Error behaviours of navigation parameters during the GPS blockage

\section{Real Data Analysis}

\subsection{Data acquisition and processing}

To analyse the influence of vehicle dynamics and trajectory on the integrated GPS/INS system performance, kinematic experiments were carried out in Clovelly Bay Car park, Sydney, on the $24^{\text {th }}$ and $25^{\text {th }}$ of March 2003. The INS used in this research was the Boeing C-MIGITS II system, which is considered to be a tactical-level

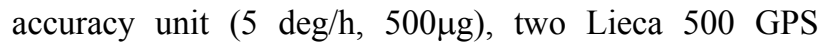
receivers were used at both the base and rover (vehicle) stations. During the data acquisition, raw INS and GPS measurements were recorded at $100 \mathrm{~Hz}$ and $1 \mathrm{~Hz}$, respectively, and there were 6 visible satellites (above the cut-off angle of $15^{\circ}$ ).

The C-MIGITS functions as an integrated GPS (MicroTracker single board)/IMU navigation system, calculating a Kalman-filtered navigation solution in realtime. In this study, the raw INS and GPS data from the Leica 500 receiver were processed using an in-house software package - the modified version of the AIMS ${ }^{\mathrm{TM}}$ navigation processing software (Grejner-Brzezinska et al, 1998a \& b; Lee et al, 2002). 


\subsection{Vehicle Dynamics Influences in the Filter estimation}

In order to study the influence of vehicle dynamics and trajectory on the estimation of the error states that have poor observability (e.g., horizontal accelerometer biases and heading error), four experiments were carried out with controlled-trajectories. Fig. 8 depicts the vehicle trajectories and dynamics during manoeuvring. For convenience, each of the trajectories is named as 'Circle', 'Line', 'Rectangle', and 'S-turn'. Note that the vertical dynamics $(\mathrm{Vd})$ are very low compared with those of the horizontal components ( $\mathrm{Vn}$ and $\mathrm{Ve}$ ).

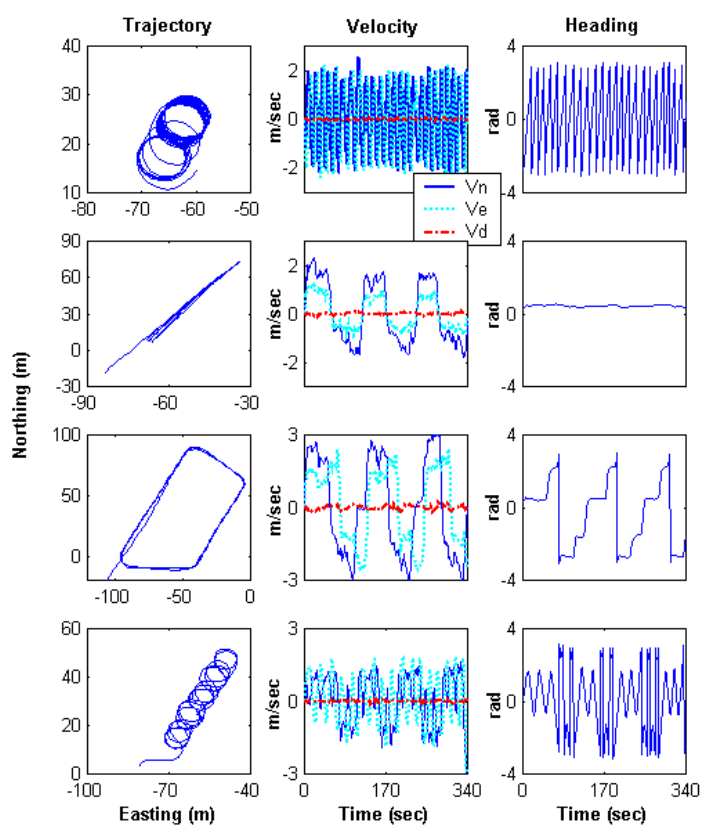

Figure 8 Vechicle trajectories and dynamics during the tests

Fig. 9 and 10 show the Root-Mean-Square (RMS) errors in horizontal accelerometer biases and heading error estimation, indicating the different vehicle dynamic contributions to the Kalman filter estimation procedure. The values were obtained from the diagonal components of the covariance matrix. It can be seen from these results that the filter estimation precision is improved by steadyturn manoeuvres (i.e., 'Circle', 'Rectangle', and 'S-turn'), when compared with the constant-velocity manoeuvre (e.g., 'Line'). This improvement can also be verified from the 'Rectangle' results showing that the filter precision is considerably increased when the vehicle makes its first right angle turn. In addition, the results in Figs. 9 and 10 show that the 'S-turn' provides the best filter estimation performance among the four trajectories considered in these tests. This is due to reversing of the vehicle's lateral acceleration that occurs in the 'S-turn' manoeuvre (Porzt and Bar-Itzhack, 1981). Note that the precision difference between the S-TURN and LINE cases is about $200 \mu g$ in horizontal acceleration and 2.5 arc-minutes in heading error estimation at the last epoch (see Figures 9 and 10).
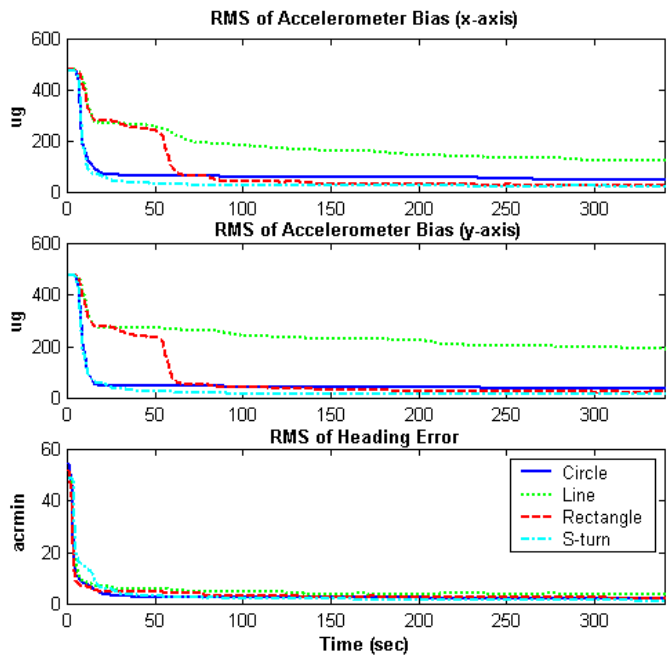

Fig. 9 RMS errors for horizontal accelerometer bias and heading error estimation
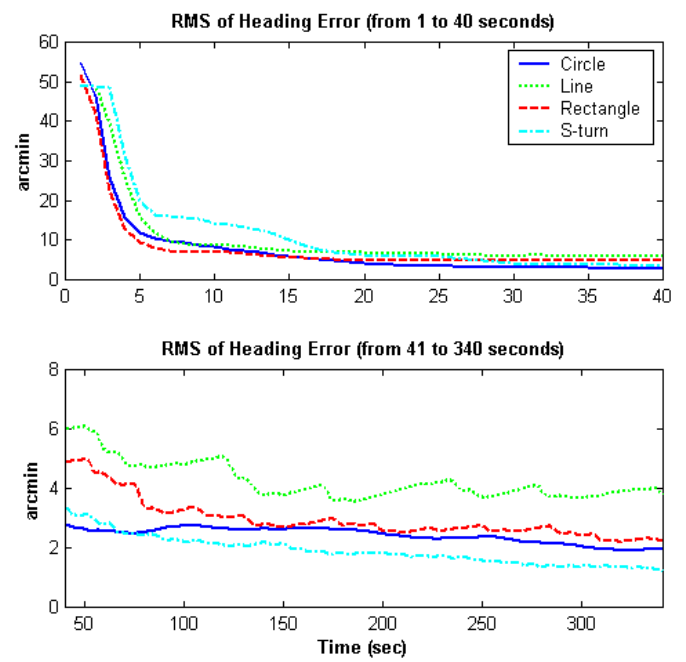

Fig. 10 Magnified results for heading RMS error in Fig. 9

\subsection{Navigation performance during GPS blockage}

Two tests were conducted to investigate the effect of vehicle dynamics on navigation error estimation in the Kalman filter during a GPS outage. The scenarios for the two tests are as follows (Fig. 11):

- The vehicle remained in static mode for a duration of 600 seconds (initialisation) before moving in circles within the same trajectory for 420 seconds;

- The vehicle stayed in static mode for a duration of 600 seconds (initialisation) before moving in circles within the same trajectory for 340 seconds, then in a straight line at a constant speed for 80 seconds. 
The GPS blockage was simulated for the last 80 seconds. Fig. 12 depicts test results showing error growth of navigation parameters during the GPS blockage. There is no abrupt error growth for the initial few tens of seconds, immediately after the outage. This seems to be due to the navigation and sensor errors being well calibrated during the first cycle with precise double-differenced carrier phase measurements. Otherwise, the results would be different from those presented. As seen from Fig. 12, the navigation parameter errors in the case of 'Circle' increase more rapidly than those in the 'Line' case during the simulated GPS blockage (i.e. stand-alone INS). Hence, highlighting that the integration filter performance is strengthened when continuously updated by external GPS measurements. This may be due to the fact that the equilibrant relationship among error parameters quickly deteriorates, the level of which is dependent on the magnitude of the vehicle dynamic change during the GPS blockage.
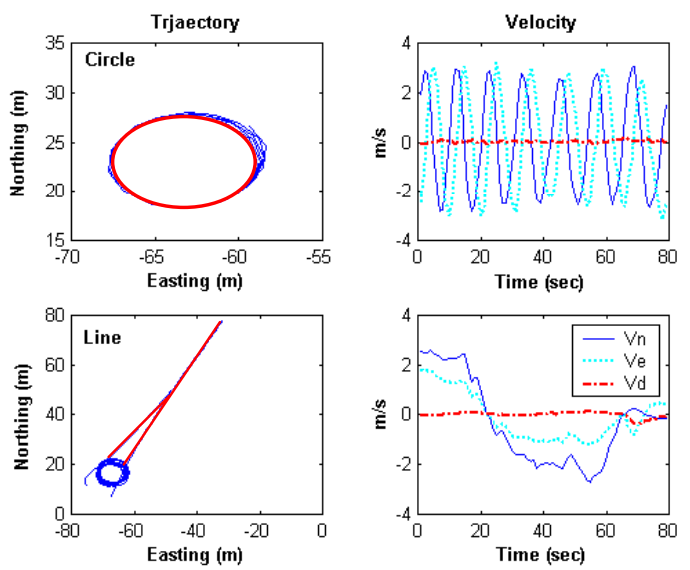

Fig. 11 Vehicle trajectories and velocities during the GPS blockage
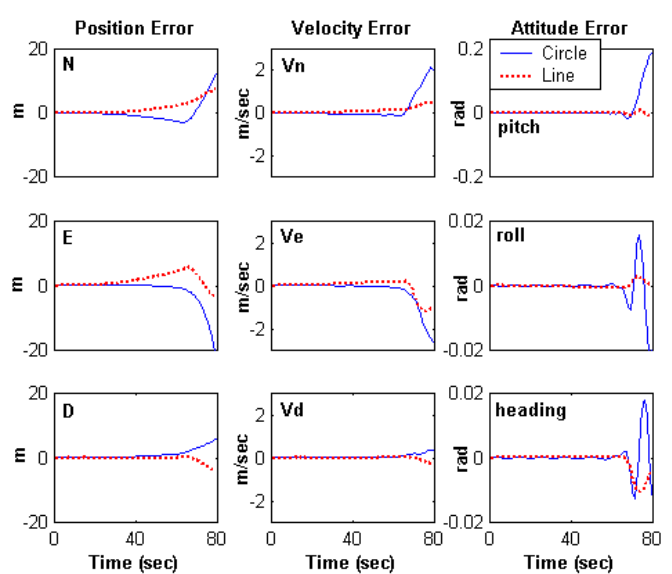

Fig. 12 Error growth of navigation parameter during the GPS blockage

\section{Concluding Remarks}

The impact of the vehicle trajectories and dynamics on the performance of the integrated GPS/INS system has been investigated in this paper through both covariance simulation and real data analyses. When the GPS measurements were used for all the data processing (no signal blockages), the results have showed that (a) vehicle dynamics affect the Kalman filter initialisation time and estimation performance, especially for the heading component; (b) the higher the dynamic changes in the lateral direction, the shorter the initialisation time; (c) the S-turn shaped trajectory provided the quickest filter initialisation of the four trajectories considered in these tests. Furthermore, throughout these simulations the performance of heading error estimation of the 'Circle', 'Rectangle' and 'S-turn' becomes similar, and the 'Circle' trajectory provides the best performance in the horizontal accelerometer biases. On the other hand, when GPS signal blockage was simulated, relatively high dynamic changes degrade the system performance; thus resulting in the rapid growth of the navigation errors.

\section{Acknowledgements}

The second author (HKL) is supported in his $\mathrm{PhD}$ research by a Scholarship funded by the Kwanjeong Educational Foundation of Korea.

\section{References}

Bar-Itzhac IY and Porat B (1980) Azimuth observability enhancement during Inertial Navigation System in-flight alignment, AIAA Journal of Guidance, Control \& Dynamics, vol. 3, no. 4, 337-344.

Bar-Itzhack IY and Berman N (1988) Control theoretic approach to inertial navigation system. AIAA Journal of Guidance, Control \& Dynamics, vol. 11, 237-245.

$\mathrm{Da} \mathrm{R}$, Investigation of a Low-Cost and High-Accuracy GPS/IMU System, Proceedings of ION National Technical Meeting, Santa Monica, California, 14-16 January, 1997, 955-963.

Farrell RA and Barth M (1988) The Global Positioning System \& Inertial Navigation, McGraw-Hill companies, Yew York, 340pp.

Greenspan RL (1996) GPS and Inertial Integration, Parkinson $\mathrm{B}$ and Spilker JJ (eds), Global Positioning System: Theory and Applications, vol. 2, chapter 7, American Institute of Aeronautics and Astronautics, Inc., Washington.

Grejner-Brzezinska D, Da R and Toth C (1998) GPS Error Modeling and OTF Ambiguity Resolution for HighAccuracy GPS/INS Integrated System, Journal of Geodesy, vol. 72, 626-638.

Grejner-Brzezinska D, Da R \& Toth C (1998) Positioning accuracy of the Airborne Integrated Mapping System, 
Proceedings of ION National Technical Meeting, Long Beach, California, 21-23 January 1998, 713-721.

Ham FM and Brown RG (1983) Observability, Eigenvalues and Kalman filtering, IEEE Transactions on Aerospace and Electronic Systems, vol. 19, no. 2, 269-273.

Hong S, Lee MH, Rios J and Speyer JL (2000) Observability Analysis of GPS Aided INS, Proceeding of ION GPS 2000, 19-22 September, 2000, Salt Lake City, UT, 26182624.

Kwon JH and Jekeli C (2001) A New Approach for Airborne Vector Gravimetry Using GPS/INS, Journal of Geodesy, vol. 74, 690-700.

Lee HK, Wang J and Rizos C (2002) Kinematic Positioning with an Integrated GPS/Pseudolite/INS, Proceedings of 2nd Symp. on Geodesy for Geotechnical \& Structural Applications, Berlin, Germany, 21-24 May, 314-325.

Maybeck PS (1979) Stochastic Models, Estimation, and Control : Volume 1, Academic Press, Inc., 423pp.

Porat B and Bar-Itzhac IY (1981) Effect of acceleration switching during INS in-flight alignment, AIAA Journal of Guidance, Control \& Dynamics, vol. 4, no. 4, 385-389.

Savage PG (2000) Strapdown Analytics : Part II, Strapdown Associates, Inc., 650pp.

Wang, J., Lee, H.K. and Rizos, C (2003) GPS/INS integration: A performance sensitivity analysis, Wuhan University Journal of Nature Sciences, Vol. 8, pp.81-94. 